\title{
A comparison between aeroacoustic source mapping techniques for the characterisation of wind turbine blade models with microphone arrays
}

\author{
Gianmarco Battista ${ }^{1}$, Marcello Vanali ${ }^{2}$, Paolo Chiariotti ${ }^{3}$, Paolo Castellini ${ }^{1}$ \\ ${ }^{1}$ Università Politecnica delle Marche, Via Brecce Bianche, 60121 Ancona, Italy \\ 2 Università di Parma, Parco Area delle Scienze, 43124 Parma, Italy \\ 3 Politenico di Milano, Via Giuseppe La Masa, 20156 Milano, Italy
}

\begin{abstract}
Characterising the aeroacoustic noise sources generated by a rotating wind turbine blade provides useful information for tackling noise reduction of this mechanical system. In this context, microphone array measurements and acoustic source mapping techniques are powerful tools for the identification of aeroacoustic noise sources. This paper discusses a series of acoustic mapping strategies that can be exploited in this kind of applications. A single-blade rotor was tested in a semi-anechoic chamber using a circular microphone array. The Virtual Rotating Array (VRA) approach, which transforms the signals acquired by the physical static array into signals of virtual microphones synchronously rotating with the blade, hence ensuring noise-source stationarity, was used to enable the use of frequency domain acoustic mapping techniques. A comparison among three different acoustic mapping methods is presented: Conventional Beamforming, CLEAN-SC and Covariance Matrix Fitting based on Iterative Re-weighted Least Squares and Bayesian approach. The latter demonstrated to provide the best results for the application and made it possible a detailed characterization of the noise sources generated by the rotating blade at different operating conditions.
\end{abstract}

Section: RESEARCH PAPER

Keywords: Aeroacoustic measurements; microphone array measurements; wind turbines; acoustic source identification

Citation: Gianmarco Battista, Marcello Vanali, Paolo Chiariotti, Paolo Castellini, A comparison between aeroacoustic source mapping techniques for characterisation of wind turbine blade models with microphone arrays, Acta IMEKO, vol. 10, no. 4, article 24, December 2021, identifier: IMEKO-ACTA10 (2021)-04-24

Section Editor: Francesco Lamonaca, University of Calabria, Italy

Received July 26, 2021; In final form September 30, 2021; Published December 2021

Copyright: This is an open-access article distributed under the terms of the Creative Commons Attribution 3.0 License, which permits unrestricted use, distribution, and reproduction in any medium, provided the original author and source are credited.

Corresponding author: Gianmarco Battista, e-mail: g.battista@staff.univpm.it

\section{INTRODUCTION}

The growing interest in renewable energy sources is requesting advances on several disciplines in order to reduce technological barriers and improve energy conversion efficiency. One of the mainstream technologies is wind power. It is well evident that the worldwide installed capacity of wind energy assets is growing exponentially since early 2000's. On the one hand, this sector is grabbing the attention of many industries and research groups, on the other hand, it is going through increasing regulations. In fact, one of the critical aspects of wind turbines is noise pollution. In order to mitigate wind turbine blade noise, the identification of location and strength of aeroacoustic noise sources is mandatory. This knowledge makes it possible to improve blade profiles and design effective aerodynamic appendages, such as trailing-edge serrations. In this work, acoustic imaging techniques, based on microphone arrays [1], have been used to characterise a scale single-blade rotor, installed in a semi-anechoic chamber, in different operating conditions. The requirements of a mapping technique for this application are:

- $\quad$ the ability to deal with rotating sources;

- sufficient spatial resolution with respect to the model size to distinguish different sources on the blade;

- $\quad$ sufficient dynamic range to identify also weak sources with respect to the strongest one.

A first classification of acoustic imaging methods can be done distinguishing time domain and frequency domain approaches. Time domain approaches [2] are typically used for selectively shaping and steering the directivity of the array (e.g., directivemicrophone like behaviour). These methods can be used when 
the sources of interest are time-variant, both in terms of position and emitted noise. [3]. Frequency domain approaches are more used for characterizing the acoustic sources in terms of location and strength. Most of frequency domain techniques make use of the Cross-Spectral Matrix (CSM) estimation from microphone signals as input data. Therefore, the effect of incoherent background noise on acoustic maps can be attenuated by means of the averaging process, moreover, some methods can handle the removal of the CSM main diagonal to neglect the contribution of incoherent noise across all the array microphones (e.g., wind noise). Conventional Beamforming (CB) [1] is the most widespread frequency domain mapping technique due to its robustness and low computational cost. However, it suffers of some limitations in terms of dynamic range and spatial resolution. In literature [1][4], several advanced frequency domain mapping techniques are available that go beyond $\mathrm{CB}$ limitations and make it possible also the quantification of the noise source.

Advanced frequency domain mapping techniques are generally preferred to time domain approaches in aeroacoustic applications since they generate acoustic maps with high dynamics and fine spatial resolution [4].

In frequency domain, three categories of mapping techniques can be identified depending on how the Region of Interest (ROI) is mapped using pressure data at microphones, i.e., how the inverse operator is defined: beamforming, deconvolution, and inverse methods. The basics of different approaches for defining inverse operators is provided in the next section, while the detailed review is provided by Leclère et al. [5].

Among beamforming techniques, it is worth noticing Functional Beamforming [6], that is a variant of CB. This simple method enhances performance and flexibility of $\mathrm{CB}$ in terms of resolution and dynamics of maps. However, it is not compatible with the diagonal removal, therefore, it is not very effective in presence of a relevant background noise.

One of the most recognised deconvolution methods is DAMAS (Deconvolution Approach for the Mapping of Acoustic Sources) [7]. This method aims at retrieving actual source distribution that generated the $\mathrm{CB}$ map by solving an inverse problem. The results achievable with DAMAS are generally suitable for all demanding applications, however, the computational effort is quite high, since it requires the calculation of the array Point Spread Function (PSF), for all candidate sources in the ROI, and finding a solution of an inverse problem.

The deconvolution method named CLEAN-SC (CLEAN based on Source Coherence) [8] is currently the state of the art as aeroacoustic applications are concerned, since it has a low computational cost (just slightly higher than $\mathrm{CB}$ ) and it is very effective in generating maps with high dynamics. The main drawback of CLEAN-SC is the spatial resolution in separating sources close together, since it has the same limitations of CB.

Lastly, inverse methods such as Generalized Inverse Beamforming [9], Bayesian Approach to sound source reconstruction [21], Equivalent Source Method [10] and Covariance Matrix Fitting (CMF) [11] aims at retrieving the complete source map at once, thus being capable of accounting for interaction between sources. However, dealing with underdetermined and ill-posed problems, they require reliable regularization techniques (e.g. Empirical Bayesian Regularization [12]).

The application of frequency domain approaches requires a stationary acoustic field that is not the case of a rotating source viewed by a static array. The Virtual Rotating Array (VRA) approach [13] has been adopted in this work to fulfil the requirement of a static source field and enable the application of any frequency domain mapping technique.

Three methods have been chosen for this test case: $\mathrm{CB}$ as baseline, CLEAN-SC since it is a reference technique for aeroacoustic source mapping and CMF based on Iterative Reweighted Least Squares and Bayesian approach (CMF-IRLS) [14]. A comparison between results obtained with frequency domain techniques is performed. Finally, the characterisation of the wind turbine blade model at different operating speed with CMF-IRLS is provided.

\section{THEORETICAL BACKGROUNDS OF ACOUSTIC SOURCE MAPPING}

The direct and the inverse acoustic problem formulation can be described as a linear problem, as frequency domain approaches are concerned. Consider a set of $N$ elementary sources (monopoles, dipoles etc.) whose complex coefficients are collected in the vector $\boldsymbol{q}$, and a set of $M$ receiver locations where the acoustic pressure is evaluated and collected in the complex vector $\boldsymbol{p}$. The discrete acoustic propagator $\boldsymbol{G}$ is a complex $M$-by- $N$ matrix that encodes the amplitude and phase relationships between the sources and the receivers, for a given frequency. The direct acoustic problem regards the calculation of pressures at receiver location (effects), given the source strengths (causes) and the acoustic propagator:

$$
\boldsymbol{G} \boldsymbol{q}=\boldsymbol{p} \text {. }
$$

This is a mathematically well determined problem with a unique solution. Conversely, the calculation of source strengths (causes) from observed pressures (effects), for given $\boldsymbol{G}$, represents the inverse acoustic problem. Solving this problem is not trivial, due to its ill-posed nature. In fact, the existence, the uniqueness, and the stability of the solution are not guaranteed [15]. The solution of inverse problems can be expressed as:

$$
\widehat{\boldsymbol{q}}=\boldsymbol{H} \boldsymbol{p},
$$

where, $\boldsymbol{H}$ is the inverse operator, that can assume different forms depending on the chosen approach. It is then clear that the source strengths $\widehat{\boldsymbol{q}}(\boldsymbol{H})$ can be only estimated. Moreover, this estimation strongly depends on a priori assumptions made with respect to the acoustic propagator and the pressure data $\boldsymbol{p}$ measured. Both direct and inverse problems can also be written in their quadratic form:

$$
\boldsymbol{G} \boldsymbol{Q} \boldsymbol{G}^{H}=\boldsymbol{P}
$$

$$
\widehat{\boldsymbol{Q}}=\boldsymbol{H} \boldsymbol{P} \boldsymbol{H}^{H},
$$

where the superscript ${ }^{H}$ denotes the conjugate transpose operator, $\boldsymbol{Q}$ and $\boldsymbol{P}$ are source and pressure Cross-Spectral-Matrix (CSM) respectively.

Beamforming approaches solve a scalar inverse problem, i.e. each potential source strength in the region of interest is estimated independently from the others. Beamforming inverse operators $\boldsymbol{h}_{n}$, named steering-vectors, are calculated as function of the direct propagator columns $\boldsymbol{g}_{n}$ and act as spatial filter, whose properties depend on their formulation [16]. Beamforming techniques are widely used for its simplicity and robustness. However, sound source quantification is not possible, unless dedicated integration techniques are applied [17]. 
Deconvolution methods have been developed to overcome beamforming limitations, since array spatial response, i.e. the Point Spread Function (PSF), affects the acoustic map retuned by beamformers. Deconvolution methods aim at retrieving the actual source distribution that generated the beamforming map removing the PSF effect.

In opposition to beamforming approach, inverse methods aim at estimating all potential sources together, hence, accounting also for interaction between sources. Frequently, this entails the solution of heavily under-determined problems, since the number of microphones (equations) is much lower than the number of potential sources in the region of interest (unknowns). Another issue with inverse methods is to adopt a robust regularization mechanism that is capable to estimate the proper amount of regularization depending on the specific problem and the measured data. Also, the choice of the ROI and its discretization with elementary sources plays a crucial role in retrieving an accurate solution. Although the complexity of these methods, the advantages justify their application.

\section{MATERIALS AND METHODS}

The object of study of this paper is a scale blade model, having a radius of $1.5 \mathrm{~m}$. The design of this model is targeted to small wind turbines, that are defined by the standard IEC61400 as the ones having the rotor spanning up to $200 \mathrm{~m}^{2}$ (about $16 \mathrm{~m}$ diameter). Figure 1 shows a simplified scheme of the experimental setup installed in the semi-anechoic chamber of Università Politecnica delle Marche. The single-blade rotor must reach $650 \mathrm{rpm}$ to operate at nominal conditions in terms of aerodynamic angle of attack and wind speed at tip. The latter corresponds to $102 \mathrm{~m} / \mathrm{s}$ or $367.2 \mathrm{kph}$ which is typical for small wind turbines.

The single-blade rotor is placed at a distance of $3 \mathrm{~m}$ in front of the circular microphone array, which has 40 equally spaced 1/4" microphones (B\&K Array Microphone Type 4951) installed on a circumference of $D=3 \mathrm{~m}$ diameter. The centre of the array is aligned with the rotation axis and the microphone plane is parallel to the blade rotation plane. An asynchronous electric motor, controlled by an inverter, drives the single-blade rotor at desired angular speed. The motor has been also equipped with an incremental encoder for measuring the angular position of the blade. All sensor signals have been synchronously acquired at $102.4 \mathrm{kSamples} / \mathrm{s}$ for each channel (microphones and encoder). Each acquisition lasts for $7.5 \mathrm{~s}$ and is performed at constant angular speed of the rotor.

Realisation of acoustic maps for moving sources usually requires time domain beamforming, since the distances from microphones to focus points constantly change over time, thus requiring time-depending delays for focusing the array. However, aeroacoustic applications require maps with high dynamic range and fine spatial resolution which is achievable only with more sophisticated frequency domain approaches. Most of them use the microphone CSM as input data to provide the acoustic map. When dealing with stochastic signals, such as aeroacoustic noise, the cross-spectra must be averaged over several time snapshots to get meaningful spectral estimation and to reduce the effect of uncorrelated background noise. The averaging process requires the sources to be stationary in time and space with respect to the array. However, pressure signals of the rotating blade, acquired with the static circular array does not fulfil these conditions, in fact, the source-microphone relative position changes over time.

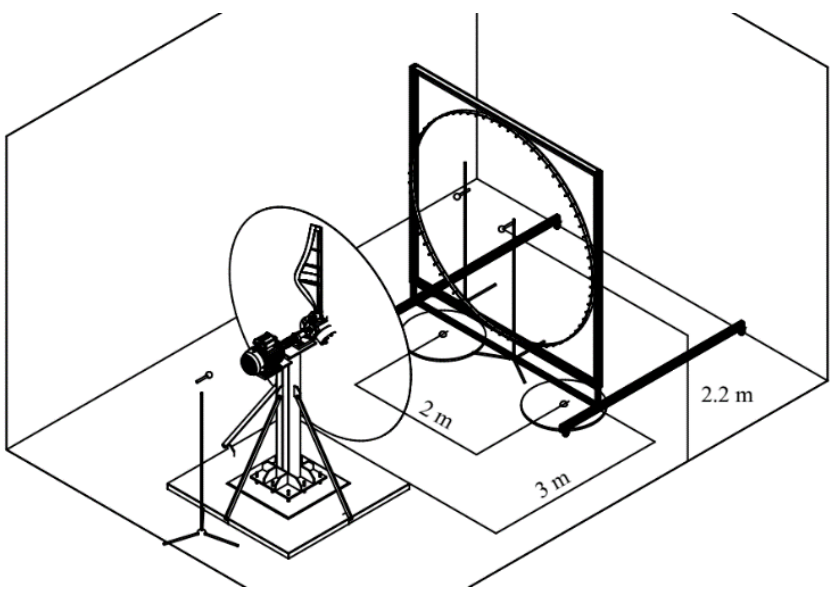

Figure 1. Scheme of the set-up in the anechoic chamber.

In order to face this aspect, the Virtual Rotating Array (VRA) [13] approach has been adopted to turn sound pressure signals, recorded with the static physical array, into signals of a virtual array that is rotating synchronously to the blade. In this way, the blade appears in a fixed position with respect to the VRA, thus making it possible to adopt any frequency domain imaging technique. The simplest realization of VRA requires a circular array, that must be parallel and co-axial with the rotor, and the knowledge of the instantaneous angular position of the blade. When a rotating array is used (both physical and virtual), the medium does not rotate at the same speed, therefore, it appears to rotate from the perspective of VRA. The acoustic propagator assumed for calculations of acoustic maps must consider the propagation of acoustic waves through a rotating flow field to obtain meaningful results.

\subsection{Virtual Rotating Array}

The working principle of VRA relies on the transformation of the pressure signals $p_{m}(t)$ recorded by the physical array into pressure signals $p_{m v}(t)$ as if they were recorded by microphones virtually rotating. The virtual array has the same layout of the physical one, thus having $M=40$ microphones equally spaced along the circumference. The position of a virtual microphone, rotating on the same circumference of the physical array, does not correspond to the position of any physical microphone most of the time, but its signal can be estimated by means of spatial interpolation of signals recorded on the array circumference. The instantaneous position of each virtual microphone is determined from the angular position of the rotor $\phi(t)$. Calculation of pressure value for each sample of a virtual microphone signal requires the identification of which pair of physical adjacent sensors must be selected for the interpolation. These are identified by the indexes $m_{l}$ and $m_{u}$, that are function of time $t$ and the virtual microphone index $m v$ :

$$
\begin{aligned}
& m_{l}(m v, t)=\left\lfloor m v+\frac{\phi(t)}{\alpha}-1\right\rfloor \bmod M+1 \\
& m_{u}(m v, t)=\left\lfloor m v+\frac{\phi(t)}{\alpha}\right\rfloor \bmod M+1,
\end{aligned}
$$

where, $[\cdot]$ is the floor function and $\alpha=2 \pi / M$ is the angular spacing between sensors. Once selected the pair of microphones for spatial interpolation, for each $t$ and $m v$, the value of virtual signal sample $p_{m v}(t)$ is calculated as the weighted sum of the samples of physical microphones 


$$
p_{m v}(t)=p_{m_{l}} s_{u}+p_{m_{u}} s_{u},
$$

being the weights determined as

$$
\begin{aligned}
& s_{u}(t)=\frac{\phi(t)}{\alpha}-\left\lfloor\frac{\phi(t)}{\alpha}\right\rfloor \\
& s_{l}(t)=1-s_{u}(t) .
\end{aligned}
$$

The signals $p_{m v}(t)$ obtained with this procedure can be used to estimate the microphone CSM.

\subsection{Processing techniques applied to the case of study}

The instantaneous angular position of the rotor $\phi(t)$ is retrieved from the encoder signal and it is used to calculate VRA time histories. The region of interest has been defined as a rectangular area of $1.80 \times 0.90 \mathrm{~m}$, positioned on the rotor plane, i.e. at $3 \mathrm{~m}$ from the array plane. From VRA point of view, this area contains the blade and the hub of the rotor. The rectangular area has been discretised with a grid of monopoles with $0.02 \mathrm{~m}$ step, thus having 4186 potential sources. The acoustic propagator from monopoles to microphones is modelled with pressure to pressure free-field propagator [14]. Geometric linear distances are replaced by the actual propagation distances, calculated considering the rotating flow field. For this purpose, the angular velocity can be assumed constant during each measurement since the fluctuations are negligible with respect to the mean value. Propagation distances have been calculated with the Acoular software [18].

As stated in the introduction, three frequency domain acoustic mapping techniques are applied to the case of study, exploiting the VRA signals: CB, CLEAN-SC and CMF-IRLS. The application of $\mathrm{CB}$ is intended here as the baseline performance of acoustic imaging techniques. The deconvolution with CLEAN-SC requires to choose only a single parameter, the loop gain, which is set here to $\varphi=0.6$. Lastly, CMF-IRLS, which belongs to the branch of inverse methods, is chosen since its fully capable to deal with spatially extended sources which is a source configuration highly and naturally expected in this application. The CMF-IRLS is used to map the full CSM, without any decomposition, and the sparsity constraint on the solution is enforced by setting $p=1$. A priori information is injected in the IRLS procedure as form of spatial weighting (named "aperture function" in Bayesian Approach). The first weighting function is the CB map that eases the localisation task, since it is a rough but reliable information on source distribution. The second weighting strategy is adopted to avoid high level peaks on the map at the edge of the ROI, typical with inverse methods. Figure 2 depicts the weighting function, determined with the cosine function near all the edges, thus resulting in a cosine-tapered spatial window. Point by point product of these two weighting functions is used as total pre-weighting. For all mapping techniques, the diagonal of CSM is removed, following the common practice adopted in aeroacoustic source imaging to minimise the effect of background noise.

\subsection{Considerations on measurement uncertainty}

A reference metrological analysis of acoustic beamforming measurements has been conducted by Castellini and Martarelli in [19], where a Type B approach, based on an analytical model, was adopted to assess how the uncertainty on input quantities affects the localisation and quantification uncertainties. Instead, MerinoMartínez et al. [17] investigated the accuracy of different mapping methods in aeroacoustic applications. In this paper, the

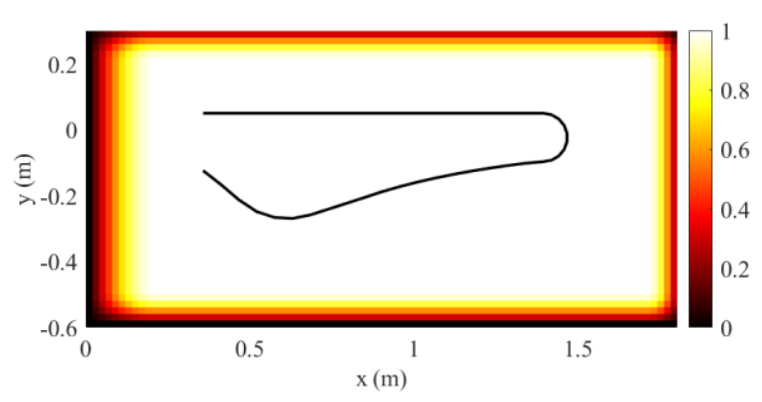

Figure 2. Arbitrary weighting function for CMF-IRLS with respect to the blade geometry.

focus is on the identification of sources of noise, rather than the absolute level. Therefore, acoustic maps must provide an estimation of source locations and their relative level. From this consideration, the target accuracy of the measurement procedure should be sufficient to distinguish different noise sources on the blade.

The resolution of mapping grid has been chosen to be compatible with the blade size and guarantee at least 2.5 potential sources for each wavelength, fulfilling the guideline provided in [20] for inverse problems (CMF-IRLS). The smallest wavelength of analysis is about $0.076 \mathrm{~m}$ (the exact value depends on the actual speed of sound). As regards beamforming-based techniques, the steering vector formulation chosen provides the correct source location at the expense of source level ("Formulation IV" described in [16]).

In array measurements, one of the most important aspects is the uniformity of frequency response of all microphones, rather than the absolute quality of the sensors. In fact, the array microphones B\&K Type 4951 are specifically designed for array applications, since they are phase-matched. The nominal sensitivity of this type of microphones is $6.3 \mathrm{mV} / \mathrm{Pa}$ (ref. 250 $\mathrm{Hz}$ ). All microphones were calibrated with the Pistonphone B\&K Type 4228, which provides a sine wave at $251.2 \mathrm{~Hz} \pm 0.1$ $\%$ and $124.0 \pm 0.2 \mathrm{~dB}$ SPL, hence the individual sensitivity was measured for each sensor. Field-calibration was performed before starting the measurement campaign. The technical specifications for free-field frequency response (ref. $250 \mathrm{~Hz}$ ) are:

$\pm 1 \mathrm{~dB}$, from $100 \mathrm{~Hz}$ to $3 \mathrm{kHz}$,

$\pm 3 \mathrm{~dB}$, from $3 \mathrm{kHz}$ to $10 \mathrm{kHz}$.

As regard the phase-matching, the manufacturer guarantees the following specifications with respect to a factory reference:

$\pm 3^{\circ}$, from $100 \mathrm{~Hz}$ to $3 \mathrm{kHz}$,

$\pm 5^{\circ}$, from $3 \mathrm{kHz}$ to $5 \mathrm{kHz}$.

The relative positions between the microphones in the array represents another source of uncertainty, which affects amplitude and phase of measured pressure. In fact, a mismatch between nominal and actual sensor locations induces an error (for each microphone) in the spatial sampling of the acoustic field, in terms of amplitude and phase. This has an influence on the quality of the maps since the nominal layout is used for calculations. However, the uncertainty on amplitude and phase, caused by frequency responses and sensor arrangement, can be considered random across the sensors, hence it can be assumed as spatial white noise. It is possible to consider this as a sort of "array noise", which is averaged across the microphones and the mean value tends to zero, as the number of sensors increases. Therefore, the source levels in the map are not significantly affected from the array noise. Instead, the standard deviation 
quantifies the level of the array noise. An accurate estimation of this parameter is rather difficult in practice, but the overall effects can be assessed comparing the degradation of array spatial response and dynamics of $\mathrm{CB}$ map with respect to the ideal condition.

An experimental test was conducted with a point source emitting a sine wave at $2 \mathrm{kHz}$ and positioned on the rotor hub (aligned with the rotation axis). A sine wave is used to have a high signal-to-noise ratio with respect to environmental acoustic background noise. The CB map obtained from this experiment is compared with the map obtained in ideal conditions with simulated data. A visual inspection of the maps reveals if the degradation of performance, due to the microphone response and sensor positioning uncertainty, is acceptable or not. Performance degradation occurring in this setup does not significantly affects the acoustic maps and is in line with the accuracy requirements.

Similar tests were conducted for assessing the rotor-array relative position and alignment. The rotor-array positioning is important to fulfil the stationarity of rotating sources with respect to the VRA. In addition to the test with the point source on the rotor hub, other four tests were done with the same source, but placed on the blade tip. In these tests, the rotor is placed at different angles (steps of $90^{\circ}$ ), still using a $2 \mathrm{kHz}$ sine wave. The rotor-array alignment was done with typical distance sensors, then CB maps were used to verify and correct it. From acoustic maps, the position of the test point sources is retrieved to estimate the offset and the angle between rotor and array axes. From the test with the source placed on the hub, the offset is estimated, which results to be in the same order of magnitude of grid resolution, in both horizontal and vertical directions. The other four tests were used to estimate the misalignment. The least square fitting plane is calculated from the four source positions, then the scalar product between the normal of the array plane and the normal of the rotor plane is calculated to estimate the angle between the two axes, which results to be less than $3^{\circ}$.

A final test with the same point source, placed at a radius of $0.6 \mathrm{~m}$ and the blade rotating at $100 \mathrm{rpm}$, was conducted to assess the correctness of all operations needed to map a rotating source via the VRA method. All mapping algorithms were able to correctly locate the point test source on the rotating blade using VRA signals.

The last important aspect is the estimation of the actual speed of sound to use for the calculation of the propagator and the steering vectors. With this purpose, two measures of air temperature inside the chamber were taken during microphone recordings, one at the beginning and one at the end of each acquisition. The model adopted for indirect measurement of the speed of sound $c$ is

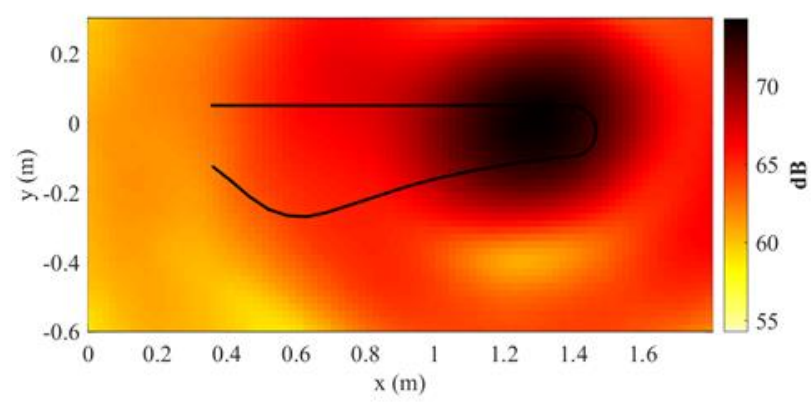

Figure 3. CB - 650 rpm. Left: $700-2500 \mathrm{~Hz}$. Right: $2500-4500 \mathrm{~Hz}$.

$$
c=331.3 \sqrt{1+\frac{T}{273.15 \mathrm{~K}}},
$$

where, $T$ is the average of initial and final air temperatures. The digital air temperature sensor has a resolution of $0.1^{\circ} \mathrm{C}$, which is sufficient for the accuracy requested in this case. Despite the importance of this parameter for the quality of the maps, little attention is often paid to this parameter [19]. In the whole test campaign, $c$ is always about $342 \mathrm{~m} / \mathrm{s}$.

\section{RESULTS}

The analysis has been performed from $700 \mathrm{~Hz}$ to $4.5 \mathrm{kHz}$. This band approximately corresponds to Helmholtz number range $6-40(\mathrm{He}=D / \lambda)$ in which the array provides adequate results. Below this range the array has very poor performance. The benchmarking of mapping techniques has been performed on the measurement acquired at the nominal operating condition of the blade, i.e. $650 \mathrm{rpm}$. For clarity of acoustic maps, the frequency range of analysis is split in two bands, where the noise generation is located in different parts of the blade.

Figure 3 shows CB maps, which are characterised by blurred sources and low dynamics. These effects, caused by the array PSF, make it difficult to identify weaker sources, since they are covered by the sidelobes of the main sources. Maps obtained with CLEAN-SC and CMF-IRLS are showed respectively in Figure 4 and Figure 5. Both methods make it possible a better localisation and they also reveal weaker sources on the blade. In fact, as expected, these methods provide higher performance in terms of spatial resolution and dynamics of acoustic maps with respect to $\mathrm{CB}$. In addition, they also provide quantitative information. The advantages of CLEAN-SC are the robustness of results, the dynamics of the maps and the low computation time. However, due to the nature of the algorithm, CLEAN-SC does not make it possible to fully represent extended sources [10]. This drawback is partially mitigated choosing a loop gain $\varphi<1$, as in this case, however, the limitation still holds. Instead, CMF-IRLS is capable to reveal the correct spatial extension of acoustic sources, but it is computationally more demanding. Despite, it is an inverse method, the maps returned by CMFIRLS do not have any source located at the edges of the mapping plane because of the pre-weighting strategy adopted. Another aspect to notice is the different source distribution returned in the low frequency range by CLEAN-SC and CMF-IRLS. Figure 4 (Left) depicts the strongest source in between the leading edge and the trailing edge, while Figure 5 (Left) shows well separated sources. This is caused by the localisation mechanism adopted by CLEAN-SC, that relies on the spatial resolution of CB. In fact,

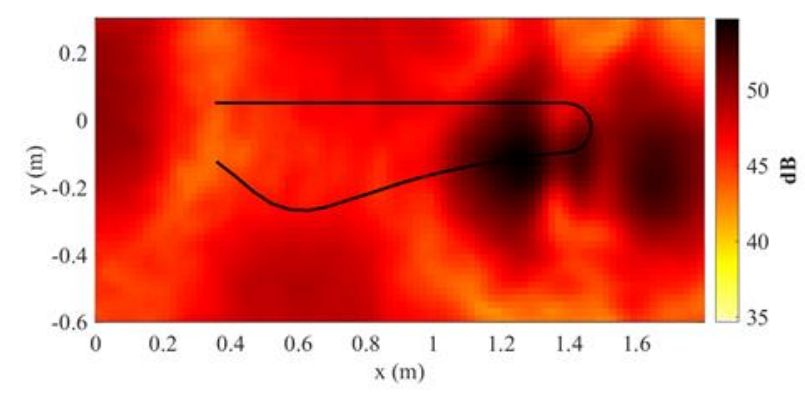




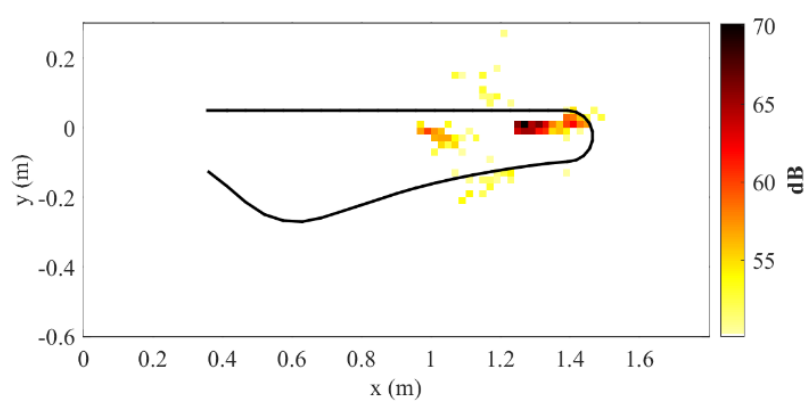

Figure 4. CLEAN-SC- $650 \mathrm{rpm}$. Left: $700-2500 \mathrm{~Hz}$. Right: $2500-4500 \mathrm{~Hz}$.

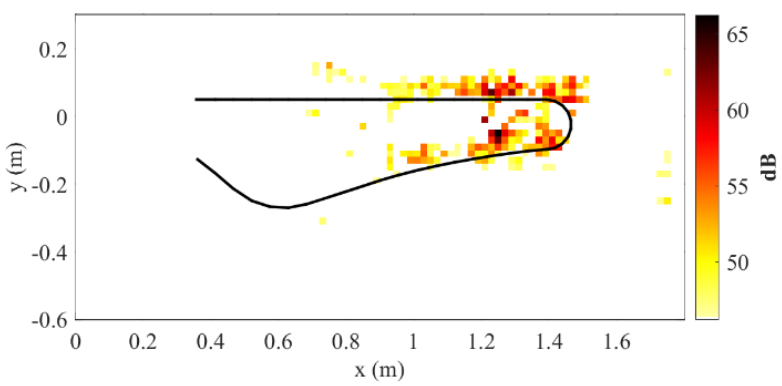

Figure 5. CMF-IRLS - 650 rpm. Left: $700-2500 \mathrm{~Hz}$. Right: $2500-4500 \mathrm{~Hz}$.

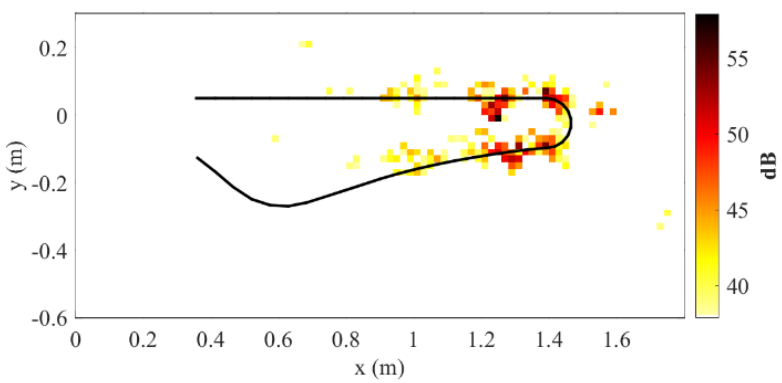

Figure 6. CMF-IRLS - 500 rpm. Left: $700-2500 \mathrm{~Hz}$. Right: $2500-4500 \mathrm{~Hz}$.

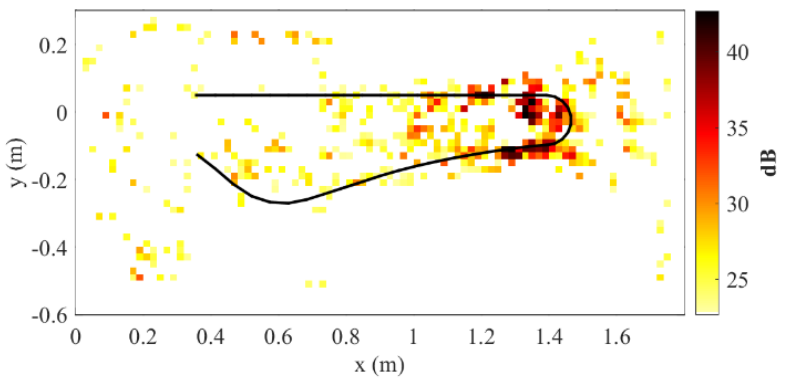

Figure 7. CMF-IRLS- $350 \mathrm{rpm}$. Left: $700-2500 \mathrm{~Hz}$. Right: $2500-4500 \mathrm{~Hz}$.

the CLEAN-SC algorithm establish that the source position is detected picking up the maximum location of the so called "dirty map", which is the output of CB at the current iteration of CLEAN-SC procedure. When two sources are closer than the mainlobe width, considering the PSF of the array at the frequency of analysis, the maximum of the total map lays somewhere in between the real sources, depending on their relative strength. This problem does not occur with CMF-IRLS, being it an inverse method, which considers all potential sources at once.
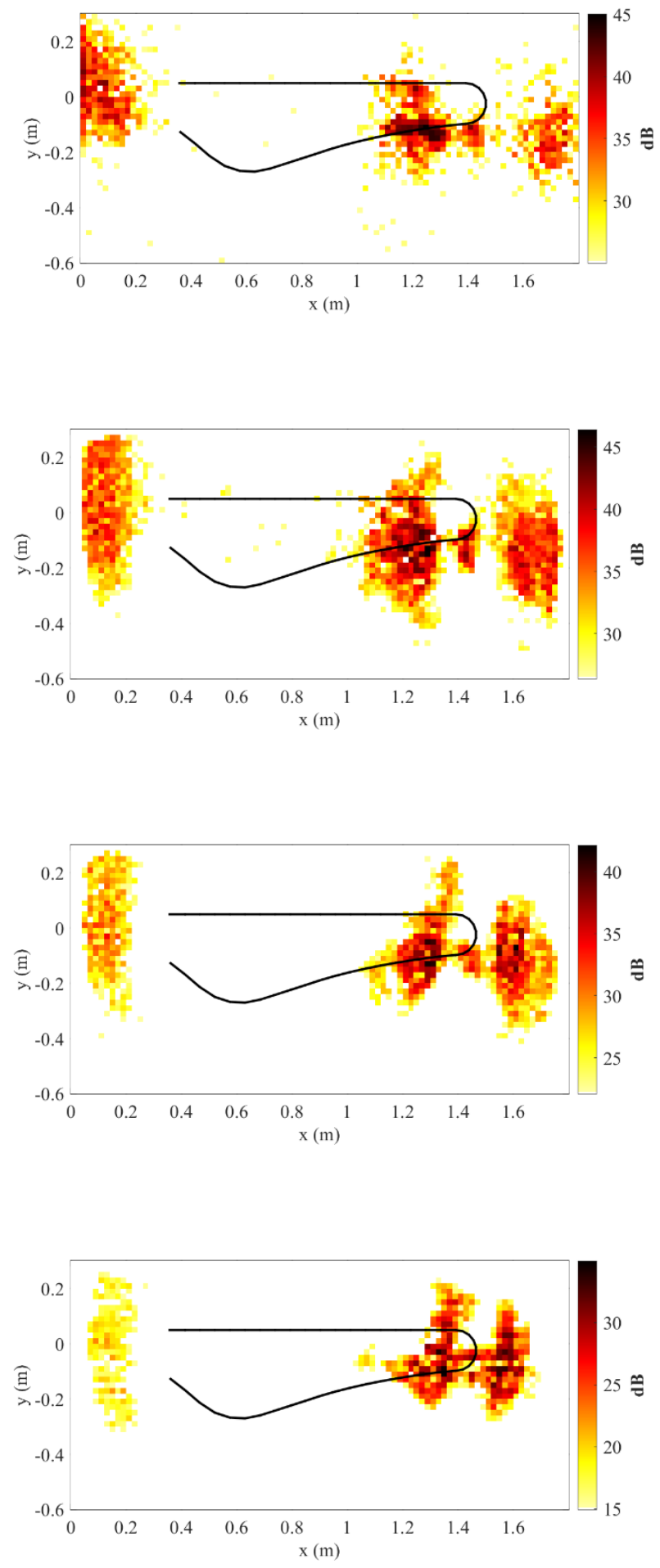

Since CMF-IRLS demonstrated to be the best performing among the methods compared in this work, it has been used for characterising the noise emission of the blade at different operating conditions. Two additional rotation speed of the rotor are tested: 500 and $350 \mathrm{rpm}$. Figure 6 and Figure 7 shows similar structures of source distribution with respect to the nominal working condition. For the lower frequency band, the noise sources are mainly in the last $0.5 \mathrm{~m}$, in the radial direction, and are quite aligned with the leading and trailing edges. For the higher band, the noise is mostly located at the tip of the blade; a 

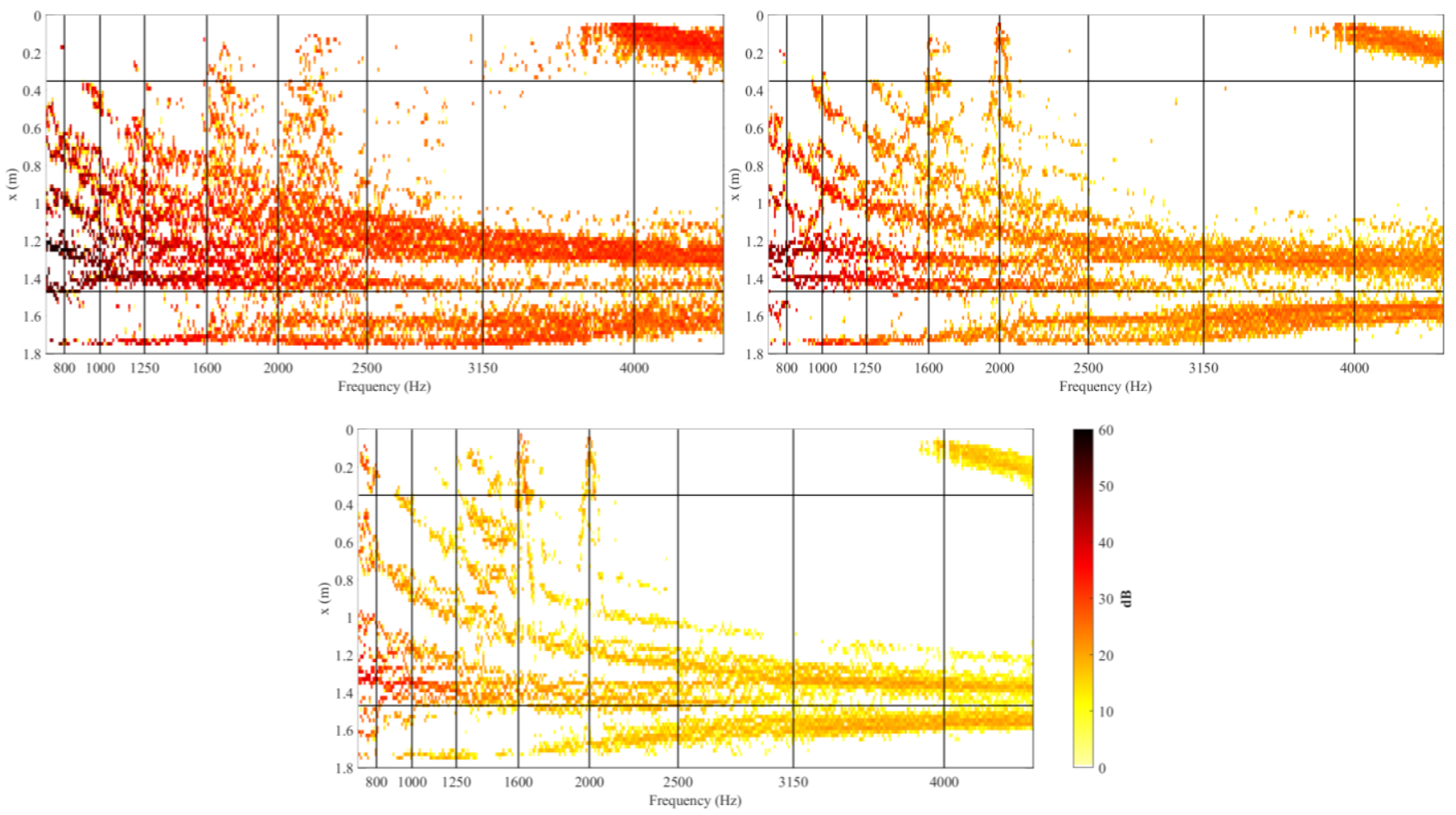

Figure 8. Chord-wise integrated maps versus frequency, CMF-IRLS. The tip and the root of the blade are represented by the horizontal black lines, while the vertical lines represent the centre of $1 / 3$ octave bands. Top left: $650 \mathrm{rpm}$. Top right: $500 \mathrm{rpm}$. Bottom: $350 \mathrm{rpm}$. All maps have the same colorscale.

source is identified at the tip location and the other two are located almost symmetrically with respect to the tip. Also some high frequency noise of the hub is visible. The extent and the level of sources decrease proportionally to the angular velocity.

In order to have an overview of how the source distribution changes with respect to frequency, a synthetic visualisation is depicted in Figure 8. This view results from the integration of the acoustic map along the chord-wise direction ( $y$ direction), therefore, it shows how the reconstructed source distribution changes with respect to frequency and radial direction $(x)$.

\section{CONCLUSIONS}

A measurement campaign has been conducted in a semianechoic chamber on a single blade rotor, for its aeroacoustic characterisation with acoustic imaging techniques exploiting microphone arrays. The strategy of VRA makes it possible to use those advanced frequency domain approaches for acoustic source mapping, that are typically required in aeroacoustic applications. The benchmarking among three methods demonstrated the advantages of CMF-IRLS versus $\mathrm{CB}$ and CLEAN-SC. The performance of CMF-IRLS is adequate for a detailed characterisation of the acoustic source distribution generated by the wind turbine blade model in different operating conditions. Therefore, this measurement technique is a powerful tool for improving the design of wind turbine blade models, since it is capable to identify aeroacoustic noise sources with high dynamic range and spatial resolution. However, the applicability is limited to models of limited size since VRA requires the array and the rotor to be aligned and co-axial.

\section{ACKNOWLEDGEMENT}

The authors would like to thank Prof. Renato Ricci of Università Politecnica delle Marche for providing the wind turbine blade model used in the measurement campaign and for the useful discussions on commenting the aeroacoustic results.

\section{REFERENCES}

[1] P. Chiariotti, M. Martarelli, P. Castellini, Acoustic beamforming for noise source localization: reviews, methodology and applications, Mechanical Systems and Signal Processing, vol. 120, 2019, pp. 422-448. DOI: $10.1016 /$ i.ymssp.2018.09.019

[2] R. Dougherty, Advanced Time-Domain Beamforming Techniques, 10th AIAA/CEAS Aeroacoustics Conference, American Institute of Aeronautics and Astronautics, 2004. DOI: $\underline{10.2514 / 6.2004-2955}$

[3] P. Sijtsma, S. Oerlemans, Location of rotating sources by phased array measurements, 7th AIAA/CEAS Aeroacoustics Conference and Exhibit, American Institute of Aeronautics and Astronautics, 2001

DOI: $10.2514 / 6.2001-2167$

[4] R. Merino-Martínez, P. Sijtsma, M. Snellen, T. Ahlefeldt, J. Antoni, C. J. Bahr, D. Blacodon, D. Ernst, A. Finez, S. Funke, T. F. Geyer, S. Haxter, G. Herold, X. Huang, W. M. Humphreys, Q. Leclère, A. Malgoezar, U. Michel, T. Padois, A. Pereira, C. Picard, E. Sarradj, H. Siller, D. G. Simons, C. Spehr, A review of acoustic imaging methods using phased microphone arrays, CEAS Aeronautical Journal, vol. 10, no. 1, Mar. 2019, Art. no. 1, DOI: $\underline{10.1007 / \mathrm{s} 13272-019-00383-4}$

[5] Q. Leclère, A. Pereira, C. Bailly, J. Antoni, C. Picard, A unified formalism for acoustic imaging based on microphone array measurements, International Journal of Aeroacoustics, vol. 16, no. 4-5, Jul. 2017, Art. no. 4-5.

DOI: $\underline{10.1177 / 1475472 \times 17718883}$ 
[6] R. P. Dougherty, Functional beamforming, 5th Berlin Beamforming Conference, 19-20 February 2014, Berlin, Germany, GFaI e.V., Berlin (2014)

[7] T. F. Brooks, W. M. Humphreys, A Deconvolution Approach for the Mapping of Acoustic Sources (DAMAS) Determined from Phased Microphone Arrays, Journal of Sound and Vibration, vol. 294, n. 4-5, July 2006, pp. 856-879.

DOI: $10.1016 /$ i.j.jsv.2005.12.046.

[8] Pieter Sijtsma, CLEAN based on spatial source coherence, International Journal of Aeroacoustics, vol. 6, no. 4, Dec. 2007, Art. no. 4. DOI: $10.1260 / 147547207783359459$

[9] T. Suzuki, L1 Generalized Inverse Beam-Forming Algorithm Resolving Coherent/Incoherent, Distributed and Multipole Sources, Journal of Sound and Vibration, vol. 330, n. 24, November 2011, pp. 5835-5851. DOI: $\underline{10.1016 / \text { i.jisv.2011.05.021 }}$

[10] G. Battista, P. Chiariotti, M. Martarelli, P. Castellini, Inverse methods in aeroacoustic three-dimensional volumetric noise source localization and quantification, Journal of Sound and Vibration, vol. 473, May 2020, p. 115208.

DOI: $\underline{10.1016 / \text { i.jsv.2020.115208 }}$

[11] T. Yardibi, J. Li, P. Stoica, L. N. Cattafesta, Sparsity constrained deconvolution approaches for acoustic source mapping, The Journal of the Acoustical Society of America, vol. 123, no. 5, 2008, Art. no. 5, DOI: $10.1121 / 1.2896754$

[12] A. Pereira, J. Antoni Q. Leclère, Empirical Bayesian Regularization of the Inverse Acoustic Problem, Applied Acoustics, vol. 97, October 2015, pp. 11-29.

DOI: $10.1016 /$ i.apacoust.2015.03.008

[13] G. Herold, E. Sarradj, Microphone array method for the characterization of rotating sound sources in axial fans, Noise Control Engineering Journal, vol. 63, no. 6, Art. no. 6, Nov. 2015. DOI: $\underline{10.3397 / 1 / 376348}$
[14] G. Battista, G. Herold, E. Sarradj, P. Castellini, P. Chiariotti, IRLS based inverse methods tailored to volumetric acoustic source mapping, Applied Acoustics, vol. 172, Jan. 2021, p. 107599. DOI: $10.1016 /$ i.apacoust.2020.107599

[15] J. Hadamard, Sur les problèmes aux dérivés partielles et leur signification physique, Princeton University Bulletin, vol. 13, pp. 49-52, 1902.

[16] E. Sarradj, Three-Dimensional Acoustic Source Mapping with Different Beamforming Steering Vector Formulations, Advances in Acoustics and Vibration, vol. 2012, 2012, no. 292695. DOI: $10.1155 / 2012 / 292695$

[17] Roberto Merino-Martínez, Salil Luesutthiviboon, Riccardo Zamponi, Alejandro Rubio Carpio, Daniele Ragni, Pieter Sijtsma, Mirjam Snellen, Christophe Schram, Assessment of the Accuracy of Microphone Array Methods for Aeroacoustic Measurements. Journal of Sound and Vibration, vol. 470, March 2020, pp. 115176. DOI: $\underline{10.1016 / \text { i.jisv.2020.115176 }}$

[18] E. Sarradj, Acoular acoustic testing and source mapping software. 2018. Online [Accessed 22 December 2021] http://www.acoular.org

[19] P. Castellini, M. Martarelli, Acoustic Beamforming: Analysis of Uncertainty and Metrological Performances. Mechanical Systems and Signal Processing, vol. 22, n. 3, April 2008, pp. 672-92. DOI: $10.1016 /$ i.ymssp.2007.09.017

[20] K. R. Holland, P. A. Nelson, The Application of Inverse Methods to Spatially-Distributed Acoustic Sources. Journal of Sound and Vibration, vol. 332, n. 22, October 2013, pp. 5727-5747. DOI: $10.1016 /$ j.jsv.2013.06.009

[21] J. Antoni, A Bayesian approach to sound source reconstruction: Optimal basis, regularization, and focusing, The Journal of the Acoustical Society of America, vol. 131, no. 4, Art. no. 4, Apr. 2012, pp 2373-2890.

DOI: $\underline{10.1121 / 1.3685484}$ 